\title{
Insuficiência Hepática Aguda da Gravidez Experiência Clinica com Sete Casos
}

\author{
Acute Liver Failure of Pregnancy - Clinical Experience with Seven Cases \\ Marcelo Perosa, Tércio Genzini, Fernando Luiz Pandullo, Paulo Jacques Gherardi Goldstein \\ César Câmara Ferreira, Marco Aurélio Perosa de Miranda, Regina Leitão
}

\begin{abstract}
RESUMO
Objetivos: avaliar a dificuldade diagnóstica, o tratamento e seu resultado em casos de insuficiência hepática aguda da gravidez.

Métodos: sete pacientes com insuficiência hepática aguda da gravidez admitidas em nosso Serviço nos últimos quatro anos foram estudadas com ênfase nos sintomas presentes, achados laboratoriais, curso clínico, complicações maternas e sobrevida fetal.

Resultados: a média de idade foi de 25, 8 anos (sendo duas primigestas) com idade gestacional média de 30,1. Destas, quatro receberam diagnóstico final de esteatose hepática aguda da gravidez e três de colestase intra-hepática da gravidez. Os principais sinais e sintomas encontrados foram: anorexia, náusea, dor abdominal, ictericia e encefalopatia. Ocorreu morte materna em dois casos: uma paciente por falência hepática enquanto aguardava órgão para transplante e outra por falência hepática, coagulopatia grave e choque hemorrágico após biópsia hepática. Uma paciente com esteatose hepática aguda evoluiu para cronicidade e encontra-se viva um ano após transplante hepático. Nos quatro casos restantes houve completa remissão do quadro com as medidas de suporte, associadas à interrupção da gravidez. As mortalidades materna e fetal foram, respectivamente, 28,6\% e 57,1\%.

Conclusões: concluiu-se, nesta experiência inicial, que a insuficiência hepática aguda da gravidez constitui evento clinico grave, de elevada mortalidade materno-fetal, e que seu pronto reconhecimento e encaminhamento para centros terciários especializados em figado, além da imediata interrupção da gestação, são fatores decisivos para o sucesso do tratamento.
\end{abstract}

PALAVRAS-CHAVE: Esteatose hepática aguda. Gravidez. Colestase. Complicações da gravidez. Óbito fetal.

\section{Introdução}

A insuficiência hepática aguda (IHA) constitui evento pouco comum, mas não raro, atingindo cerca de 2.000 pacientes todos os anos nos Estados Unidos e alcançando mortalidade de até $80 \%$ dos $\operatorname{casos}^{1,2}$.

Várias doenças hepáticas podem incidir durante a gravidez, trazendo sérias conseqüências para a mãe e o feto ${ }^{3-5}$. Segundo Riely ${ }^{6}$, as doenças hepáticas que ocorrem durante a gestação podem

Hospitais São Camilo e Santa Helena (São Paulo) Correspondência:

Marcelo Perosa

Rua Maestro Cardim 377, cj 75 - Paraíso

01323-001 - São Paulo - SP ser divididas em três grupos: 1) doenças hepáticas pré-existentes, incluindo a hepatite auto-imune e a doença de Wilson; 2) doenças hepáticas exacerbadas pela gravidez, como infecções virais e a hepatite pelo herpes simples; 3) doenças hepáticas que ocorrem apenas durante a gravidez e por ela são desencadeadas, tais como colestase intra-hepática da gravidez (CIHG), pré-eclâmpsia grave com sindrome HELLP (hemólise, elevação de enzimas hepáticas e plaquetopenia) e a esteatose hepática aguda da gravidez (EHAG).

Esta última ocorre em aproximadamente 1 de cada 13.000 gestações ${ }^{1,5}$, ao passo que a sindrome HELLP, mais prevalente, soma 1 a 6 casos em 1.000 gestações $^{5,7-9}$. A CIHG tem incidência variada conforme a região, atingindo 
prevalência elevada em países como a Suécia e Finlândia (1 a $1,5 \%)$ e Chile $(11,8 \%)$ e baixa em países como os Estados Unidos, Canadá e França, variando de 0,2 a $0,5 \%{ }^{10,11}$.

O reconhecimento precoce destas afecções, assim como o encaminhamento a centros terciários especializados em figado, é de fundamental importância para o sucesso do tratamento materno e fetal ${ }^{5,8}$.

O espectro das complicações hepáticas durante a gravidez é bastante variado, abrangendo formas de apresentação desde oligo ou assintomática até a IHA grave, com elevado risco de vida e por vezes tendo no transplante hepático seu último recurso terapêutico possivel ${ }^{4,12-14}$.

Nos últimos três anos, recebemos encaminhamento de sete gestantes com doença hepática da gravidez (DHG) de evolução clínica variada. Diante da baixa incidência, do alto risco e da escassez de estudos abordando estas complicações em nosso meio, relata-se a seguir esta experiência inicial, enfocando especialmente os aspectos clínicos, histológicos e a terapêutica adotada.

\section{Pacientes e Métodos}

Entre julho de 1997 e março de 2000, sete pacientes com DHG foram admitidas e tratadas em nosso Serviço. Em todos os casos, tratava-se de pacientes com gestação até então em curso normal que, durante o terceiro trimestre, desenvolveram rapidamente sinais e sintomas de IHA, além de alterações das provas de função hepática. Nenhuma paciente apresentava antecedentes de transfusões sangüineas, ingestão de drogas hepatotóxicas, uso abusivo de álcool ou sinais clínicos de pré-eclâmpsia. As sorologias para hepatite viral A, B, C e E foram negativas em todas as pacientes. Realizaram-se provas de função hepática e outros testes laboratoriais seriadamente desde a admissão até a total regressão dos sinais de doença hepática e de suas complicações. Todas as pacientes foram submetidas a estudo ecográfico com Doppler do figado, que se mostrou normal, afastando-se presença de complicações vasculares hepáticas ou bílio-pancreáticas. Sempre que possivel, optou-se pela realização da biópsia hepática para confirmação diagnóstica. A Tabela 1 discrimina os dados epidemiológicos e gestacionais das pacientes. Em cinco casos, as pacientes foram admitidas em nosso Serviço no periodo puerperal, encaminhadas devido às complicações hepáticas. Nos dois casos restantes, as pacientes tiveram seu parto realizado em nossa Instituição.

Tabela 1 - Dados epidemiológicos e gestacionais.

\begin{tabular}{ccccccc}
\hline Paciente & $\begin{array}{c}\text { Idade } \\
\text { (anos) }\end{array}$ & Cor & $\begin{array}{c}\text { Paridade } \\
\text { (No.) }\end{array}$ & $\begin{array}{c}\text { Início dos sintomas } \\
\text { (semanas) }\end{array}$ & Parto & Sexo do RN \\
\hline 1 & 18 & Branca & 1 & 31 & Normal & $\mathrm{M}$ \\
2 & 33 & Negra & 2 & 27 & Cesárea & $\mathrm{M}$ \\
3 & 19 & Branca & 2 & 28 & Cesárea & $\mathrm{M}$ \\
4 & 24 & Branca & 2 & 32 & Cesárea & $\mathrm{F}$ \\
5 & 35 & Branca & 2 & 32 & Cesárea & $\mathrm{M}$ \\
6 & 27 & Branca & 2 & 36 & Cesárea & $\mathrm{M}$ \\
7 & 25 & Branca & 1 & 25 & Normal & $\mathrm{F}$ \\
Mediana & 25 & - & - & 31 & - & - \\
\hline
\end{tabular}

As pacientes foram acompanhadas diariamente por nossa equipe, sendo transferidas para Unidade de Terapia Intensiva quando necessário e destinando atenção especial às medidas de suporte para encefalopatia hepática, monitorização diária da função hepática, medidas de controle da insuficiência renal, incluindo adequada hidratação e uso criterioso de diuréticos, cuidados com hipoglicemia e reposição de glicose intravenosa se necessário, evitando-se a trans- fusão de fatores de coagulação, já que estes testes constituem prova valiosa no acompanhamento da gravidade da IHA. Fez parte também do seguimento destas pacientes avaliar diariamente se as mesmas reuniam critérios de indicação de transplante hepático de urgência, situação em que tal recurso pode representar a única chance de tratamento e não deve ser postergado.

Analisamos retrospectivamente a forma de apresentação clínica e laboratorial, métodos diag- 
nósticos e recursos terapêuticos em todos os casos.

\section{Resultados}

Na Tabela 2 demonstra-se o diagnóstico final de cada caso, os métodos utilizados para a conclusão dos diagnósticos e a via de acesso para biópsia hepática quando empregada.

Os sinais clinicos e sintomas mais freqüentes estão expostos na Tabela 3. Nota-se a presença de mal-estar, náuseas, letargia, dor abdominal, icterícia e encefalopatia em praticamente todos os pacientes.
Tabela 2 - Diagnóstico final dos 7 casos de insuficiência hepática aguda na gravidez.

\begin{tabular}{cccc}
\hline Paciente & $\begin{array}{c}\text { Método } \\
\text { diagnóstico }\end{array}$ & $\begin{array}{c}\text { Via de acesso da } \\
\text { biópsia hepática }\end{array}$ & $\begin{array}{c}\text { Diagnóstico } \\
\text { etiológico }\end{array}$ \\
\hline 1 & Clínico & - & EAG \\
2 & Clínico/histológico & Percutânea & CIHG \\
3 & Clínico/histológico & Transjugular & EAG \\
4 & Clínico & - & CIHG \\
5 & Clínico/histológico & Céu aberto & EAG \\
6 & Clínico/histológico & Percutânea & CIHG \\
7 & Clínico/histológico & Céu aberto & EAG \\
\hline
\end{tabular}

EAG - Esteatose aguda da gravidez; CIHG - colestase intra-hepática da gravidez

Tabela 3 - Sinais e sintomas mais freqüentes em casos de insuficiência hepática aguda da gravidez (7 casos).

\begin{tabular}{|c|c|c|c|c|c|c|c|}
\hline Sinais e Sintomas & Caso 1 & Caso 2 & Caso 3 & Caso 4 & Caso 5 & Caso 6 & Caso 7 \\
\hline Mal-estar & + & + & + & + & + & + & + \\
\hline Náusea & - & + & + & + & + & - & + \\
\hline Vômitos & - & + & + & - & + & - & + \\
\hline Cefaléia & + & + & - & - & + & - & - \\
\hline Letargia & + & + & + & + & + & + & - \\
\hline Dor abdominal & + & + & + & - & + & + & + \\
\hline Prurido & + & - & + & + & + & + & - \\
\hline Icterícia & + & + & + & + & + & + & + \\
\hline Ascite & + & + & + & - & + & + & - \\
\hline Encefalopatia (grau) & III & II & 1 & - & IV & I & I \\
\hline
\end{tabular}

Do ponto de vista laboratorial, praticamente todas as pacientes apresentaram hipoglicemia (glicemia média de $48,7 \mathrm{mg} / \mathrm{dL}$ ), insuficiência renal (uréia média de $83,8 \mathrm{mg} / \mathrm{dL}$; creatinina média de 2,05$)$, plaquetopenia $\left(<100.000 / \mathrm{mm}^{3}\right)$ e leucocitose (média de $17.071 / \mathrm{mm}^{3}$ ), alterações estas semelhantes entre o grupo que evoluiu com CIHG ou EHAG. Entretanto, notou-se que houve maior comprometimento hepático nas pacientes com EHAG (aspartato aminotransferase média de 546,7 U/L; INR - relação internacional normalizada - média de 3,57 ) em comparação com as que apresentaram CIHG (aspartato aminotransferase média de 162,6 U/L; INR média de 2,86). Por outro lado, o grau de colestase foi semelhante entre os dois grupos de pacientes (bilirrubinas totais com média de $16,7 \mathrm{mg} / \mathrm{dL}$; gama glutamil transferase média de 127,4 U/L).

Para a resolução da gravidez foram realizadas cinco cesareanas e dois partos vaginais, sendo cinco recém-nascidos do sexo masculino e não houve gestação gemelar. Ocorreram quatro óbitos fetais, totalizando $57,1 \%$ de mortalidade fetal.

Quanto à evolução materna, seis pacientes necessitaram de internação em Unidade de Terapia Intensiva devido ao quadro de encefalopatia hepática muitas vezes associado à insuficiência renal, coagulopatia e hipoglicemia grave, sendo que o tempo de permanência das que apresentaram EHAG $(8,2$ dias em média) foi superior em relação as que cursaram com CIHG (4 dias em média). Tal observação foi semelhante ao se comparar os dias de internação hospitalar: 26,7 dias em média nos casos com EHAG e 13,7 dias naqueles com CIHG. Quatro pacientes apresentaram total restabelecimento da função hepática e receberam alta hospitalar em boas condições gerais e sem qualquer seqüela do ponto de vista hepático.

Uma paciente (caso 3), com diagnóstico de EHAG, apesar de receber alta hospitalar, nunca demonstrou total normalização da função hepática. A mesma evoluiu com quadro de desnutrição grave, piora das provas de função hepática, ascite, varizes de esôfago e achado ecográfico de hiper- 
tensão portal, caracterizando cronicidade da doença hepática. Por esta razão foi inscrita na fila para transplante hepático, realizado um ano e meio após a gestação que desencadeou a EHAG. Atualmente encontra-se no primeiro ano pós-transplante hepático, com total recuperação nutricional e da função hepática e retornando às suas atividades cotidianas.

Outra paciente (caso 5) evoluiu com piora progressiva da função hepática em questão de cinco dias mesmo após a interrupção da gestação. Devido à coagulopatia grave, apresentou hematoma intra-uterino pós-cesariana, que necessitou de histerectomia de urgência. Após esta intervenção, evoluiu com encefalopatia grau IV, necessitando de ventilação mecânica contínua, sendo então indicado o transplante hepático de urgência. Por se tratar de caso de IHA fulminante, recebeu prioridade na Central de Transplantes, tendo disponivel doador cadavérico apenas quatro dias após a solicitação. Realizou-se a retirada do figado do doador na tentativa de dar seqüência ao transplante hepático. Todavia, a paciente já se apresentava, naquele momento, instável hemodinamicamente, necessitando de drogas vasoativas em altas doses, sem condições clínicas sequer para indução anestésica. Optou-se então pela não-realização do transplante e a pacientes faleceu 24 horas após esta tentativa. O órgão retirado foi encaminhado em tempo para outra equipe que assim o utilizou em outro paciente.

A paciente No. 7 desenvolveu piora progressiva da função hepática em 5 dias, apresentando coagulopatia grave. Foi submetida a biópsia hepática a céu aberto e, na seqüência, interrupção da gravidez por parto vaginal. Após estes dois procedimentos, evoluiu com abdome agudo hemorrágico desencadeado pela coagulopatia e provavelmente relacionado a sangramento oriundo da biópsia hepática. Como não respondeu às medidas de reposição de hemoderivados e expansores, foi submetida à laparotomia de urgência, porém devido ao estado de choque hipovolêmico e falência hepática, evoluiu a óbito no $1^{\circ}$ dia pós-biópsia.

Com dois óbitos maternos (ambos em pacientes com EHAG) e quatro fetais (três destes filhos de mães com EHAG), a mortalidade materna foi de $28,6 \%$ e a fetal $57,1 \%$.

Em cinco pacientes realizou-se biópsia hepática para confirmação diagnóstica, uma por via transjugular, duas a céu aberto e duas por via percutânea após melhora das provas de coagulação. Na Figura 1, ilustra-se um dos casos de EHAG, destacando-se a presença de esteatose macro e microvesicular intensa e difusa, além de balonização hepatocelular. Na Figura 2, demons- tra-se caso de CIHG, notando-se predominantemente a presença de infiltrado inflamatório lobular, com colestase canalicular e intracelular, além de tumefação hepatocelular.

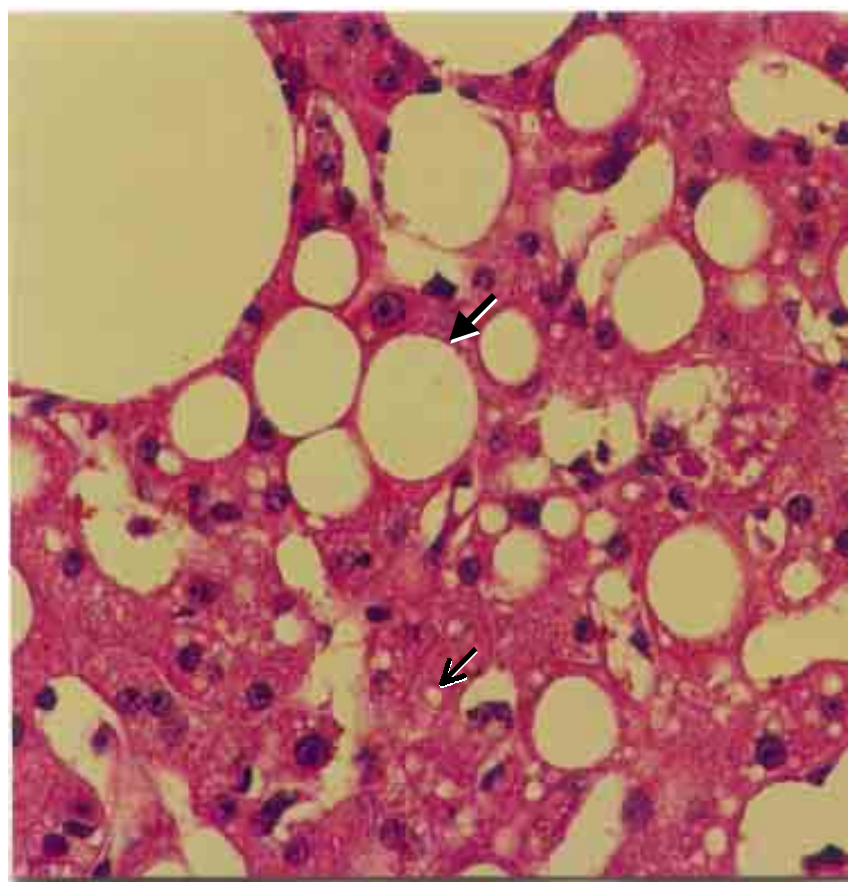

Figura 1 - Microscopia de biópsia hepática demonstrando focos de colapso centrolobular e periportal, esteatose macrogoticular (seta maior) e microgoticular (seta menor) em $90 \%$ do tecido hepático, achados característicos de esteatose hepática aguda da gestação (HE 100X).

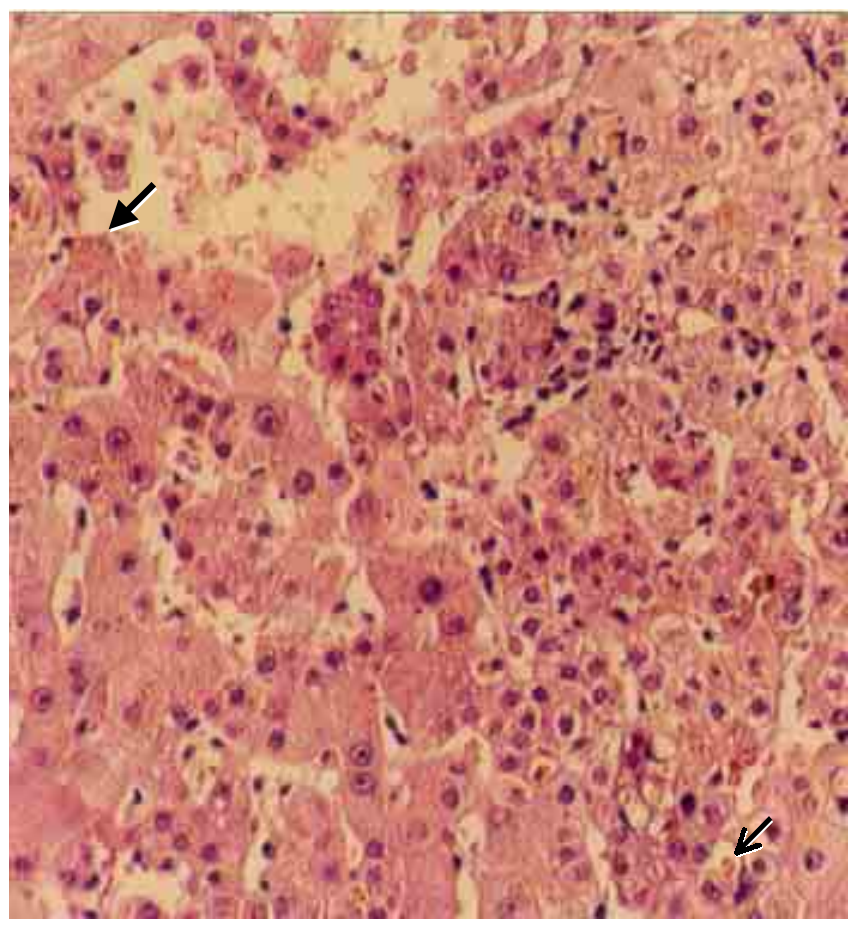

Figura 2 - Microscopia de biópsia hepática mostrando colestase intracelular (seta maior) e canalicular (seta menor), além de tumefação hepatocelular, caracterizando quadro de colestase intra-hepática da gravidez (HE 250X). 


\section{Discussão}

Poucas condições na Medicina são mais dramáticas ou devastadoras quanto a IHA. Esta abrange uma série de condições que levam a grave lesão dos hepatócitos ou necrose maciça. A perda da função hepatocitária estabelece resposta de múltiplos órgãos e a morte pode ocorrer mesmo quando a função hepática já se encontra em recuperação ${ }^{2}$.

Até a década de 1970, a IHA na gravidez apresentava evolução desfavorável, com mortalidade materno-fetal de até $85 \%{ }^{13}$. O reconhecimento precoce destes distúrbios, a pronta interrupção da gravidez e a melhora do suporte de terapia intensiva determinaram grande progresso, com redução dos índices de mortalidade materna e fetal a niveis respectivamente de 10 e $23 \%{ }^{5,15,16}$.

Dentre as patologias que mais comumente podem levar à DHG incluem-se hepatites virais, hepatites por drogas, pré-eclâmpsia, a EHAG e a $\mathrm{CIHG}^{4,6,17}$.

A EHAG, um grave distúrbio que ocorre usualmente entre a $30^{\mathrm{a}}$ e $40^{\mathrm{a}}$ semana de gestação, inicia-se geralmente com quadro gradual de anorexia, mal-estar e cefaléia. Estes sintomas inespecíficos são seguidos por vômitos, que podem ser persistentes e intensos, dor abdominal e febre. A dor abdominal pode ser difusa, epigástrica ou localizada apenas no quadrante superior direito ${ }^{1,5,14,15}$. A icterícia freqüentemente aparece vários dias após o início dos primeiros sintomas e pode ser sucedida por distúrbio de coagulação, com sangramento difuso, hipoglicemia, encefalopatia hepática, sonolência e coma ${ }^{6,8,14,16}$. Complicações associadas como insuficiência renal, pancreatite e sangramento gastrintestinal são comuns ${ }^{14,17}$. O diagnóstico definitivo da EHAG é obtido pela biópsia hepática, nem sempre exeqüivel devido aos graves distúrbios de coagulação associados ${ }^{14,17}$. As amostras de tecido hepático demonstram alterações difusas de microvesículas de gordura (esteatose) principalmente em região centrolobular à microscopia óptica ou eletrônica (Figura 1), diferentemente das alterações patológicas clássicas no figado em pacientes com pré-eclâmpsia ou sindrome HELLP ${ }^{13,14,16-18}$.

Apesar de numerosas hipóteses terem sido formuladas acerca de sua etiologia, seu mecanismo ainda não está esclarecido. A EHAG claramente não é uma doença infecciosa nem tampouco metabólica. Não há casos familiares e as gestações subseqüentes têm sido normais nas mulheres que sobrevivem à doença e posteriormente engravidam. Relata-se freqüente associação entre pré-eclâmpsia e $\mathrm{EHAG}^{6-8,14,16}$. Alguns autores sugerem até que o espectro de comprometimento do figado na pré-eclâmpsia inclua a $\mathrm{EHAG}^{19}$. Esta hipótese é reforçada também pela superposição entre as duas condições, incluindo sua maior incidência em nulíparas, partos gemelares e apresentação no terceiro trimestre da gestação ${ }^{1,5,15}$. Chama a atenção em nossa casuística a não-associação entre pré-eclâmpsia e EHAG nos casos analisados. Por se tratar de doença grave e muitas vezes fatal, o princípio básico do seu tratamento inclui o rápido diagnóstico, a interrupção imediata da gravidez e o encaminhamento precoce para serviços especializados em doenças do fíga$\mathrm{do}^{5,6,15,16,20}$. O momento adequado para este encaminhamento se dá com os primeiros sinais de encefalopatia hepática. Pode-se observar na Tabela 3 e 4 que todas as pacientes apresentavam icterícia e coagulopatia e todas, exceto uma, demonstravam ascite, encefalopatia hepática, alteração da função renal e hipoglicemia à admissão, o que caracteriza o encaminhamento tardio e a gravidade maior destes casos no momento da internação, diminuindo também a chance de sucesso do tratamento. A interrupção da gravidez deverá preferencialmente ser realizada por via vaginal, uma vez que tais pacientes cursam comumente com quadro de coagulopatia grave e as complicações hemorrágicas ocorrem em até $30 \%$ dos casos solucionados por cesarianas, o que representa fator de mau prognóstico ${ }^{5,14,15}$. Tal complicação foi observada no caso No. 5 de nossa casuística, que, após parto cesáreo, desenvolveu hematoma intra-uterino extenso e também de parede abdominal, necessitando de várias hemotransfusões e posteriormente de histerectomia de urgência, agravando ainda mais sua condição geral.

Em cerca de $2 \%$ dos casos de EHAG, as pacientes evoluem para IHA grave e irreversível, necessitando de transplante hepático de urgência ${ }^{20}$. Em nossa experiência, tal evolução foi verificada nos casos No. 3 e No. 5, solicitando-se prioridade para transplante hepático de urgência. No caso No. 3 , a paciente evoluiu com melhora parcial da função hepática enquanto aguardava pelo órgão, sendo por isto removida da lista de prioridade para transplante de figado. Apesar de incomum, evoluiu com cronicidade da doença hepática, traduzida por hipertensão portal, piora progressiva da função hepática e estado de desnutrição grave, sendo então submetida a transplante hepático com sucesso um ano e meio após a gestação. Quanto ao caso No. 5, a paciente evoluiu com rápida piora da função hepática em periodo de apenas cinco dias e após a complicação hemorrágica já referida, foi submetida a histerectomia de urgência, evoluindo, após o procedimento, para coma hepático, in- 
suficiência renal e necessidade de drogas vasoativas em altas doses, falecendo enquanto aguardava um figado a ser transplantado. Na paciente No. 7, outro caso de EHAG, realizou-se a biópsia hepática a céu aberto na vigência de coagulopatia grave. Tal fato desencadeou quadro de abdome agudo hemorrágico e, associado à IHA avançada, foi fator decisivo para o óbito. Tal biópsia foi indicada mais por questão legal e de amparo jurídico para se interromper a gestação. Esta solicitação partiu da equipe obstétrica que assistia ao caso. A evolução desta paciente reforça a idéia de que as biópsias hepáticas devem ser evitadas em condições de coagulopatia grave, devido ao elevado risco e também por não serem decisivas na conduta obstétrica. Na verdade, o fator primordial para a decisão de se interromper a gravidez é o próprio quadro clínico e laboratorial da IHA. O diagnóstico histológico é útil, mas pode ser postergado para um segundo tempo, assim que os fatores de coagulação melhorarem, como se procedeu nos casos 2 e 6.

A CIHG constitui outra doença hepática específica do período gestacional. Sua causa é ainda desconhecida, mas os resultados de estudos clinicos e epidemiológicos sugerem fatores genéticos, hormonais e exógenos ${ }^{3,4,10,11,21}$. Dentre estes, a hiperestrogenemia parece ser um dos fatores mais importantes para o aparecimento da CIHG em mulheres predispostas ${ }^{3,22}$. Geralmente manifesta-se no terceiro trimestre da gestação e com história precoce de prurido em até $70 \%$ dos casos. Apresenta evolução clínica mais branda quando comparada à EHAG, predominando a icterícia e o prurido como sintomas, sendo rara a piora da função hepática, como a presença de encefalopatia hepática e insuficiência renal ${ }^{6,8,15,16,23}$. Tal evolução também foi verificada em nossa casuística, em que estes casos (2 e 4) apresentaram evolução materna mais favorável e de rápido restabelecimento. Todavia, a CIHG é acompanhada de alto risco de prematuridade na gravidez e óbito fetal ${ }^{21,24}$. De fato, um de nossos três casos de CIHG cursou com parto prematuro de 27 semanas e óbito fetal. O tratamento da CIHG consiste em medidas que visam reduzir o grau de colestase. Entre estas, pode-se empregar o ácido ursodesoxicólico (apesar de seus efeitos colaterais materno-fetais não serem totalmente conhecidos) e a colestiramina. Recomenda-se também evitar o uso de progesterona natural em pacientes com história pregressa de colestase intra-hepática. Além disso, o uso de estrógeno oral deve ser imediatamente suspenso ao surgirem os primeiros sinais de $\mathrm{CIHG}^{3,4,22,25}$.

Devido a sua evolução mais favorável, não é obrigatória a interrupção imediata da gravidez no momento do diagnóstico, diferente da EHAG, em que pese a necessidade de controle rigoroso da vitalidade fetal devido aos riscos já comentados ${ }^{3}$.

Concluindo, a IHA na gravidez é evento raro, mas potencialmente fatal, e sua adequada condução inclui o rápido diagnóstico, interrupção da gravidez especialmente em casos de EHAG e encaminhamento precoce a centros especializados em figado, onde se possa oferecer suporte intensivo máximo a estas pacientes.

\section{SUMMARY}

Purpose: to evaluate the diagnostic difficulties, treatment and outcome in cases of acute liver failure of pregnancy.

Methods: seven patients with acute liver failure of pregnancy, managed during the past 4 years, were studied with emphasis on presenting symptoms, laboratory findings, clinical course, maternal complications and fetal outcome.

Results: the mean age was 25.8 years (two were primigravidas), and the mean gestational age at onset was 30.1 weeks. The final diagnosis was acute fatty liver of pregnancy in four cases and intrahepatic cholestasis of pregnancy in three cases. Anorexia, nausea, abdominal pain, jaundice and encephalopathy were the main clinical findings. Two maternal deaths occurred: one due to hepatic failure, while waiting for transplantation, and another due to hepatic failure associated with coagulopathy and major intraabdominal bleeding after liver biopsy. One patient with acute fatty liver of pregnancy underwent a successful liver transplantation one year ago (after chronification of her disease). The remaining four cases had complete resolution of the disorders after delivery and supportive measures. Maternal and fetal mortalities were $28.6 \%$ and $57.1 \%$, respectively.

Conclusion: from this initial experience, we conclude that acute liver failure of pregnancy is a serious disease, with high maternal-fetal mortality, and that early recognition of this illness, referral to tertiary liver centers, and prompt interruption of gestation are, together, very important for the success of the treatment.

KEY WORDS: Acute fatty liver. Cholestasis. Fetal death. Pregnancy, complications.

\section{Referências}

1. Báez-Torres A, González-Keelan C. Acute fatty liver of pregnancy. Case report and review of the literature. P R Health Sci J 1994; 13:9-12.

2. Lee WN. Acute liver failure. N Engl J Med 1993; 329:1862-72. 
3. Davidson KM. Intrahepatic cholestasis of pregnancy. Semin Perinatol 1998; 22:104-11.

4. Hunt CM, Shakara AI. Liver disease in pregnancy. Am Fam Phys 1999; 59:829-36.

5. Pereira SP, O’Donohue J, Wendon J, Williams R. Maternal and perinatal outcome in severe pregnancy related liver disease. Hepatology 1997; 26:1258-62.

6. Riely CA. Hepatic disease in pregnancy. Am J Med 1994; 96:18S-22S.

7. Dani R, Mendes GS, Medeiros JL, Péret FJ, Nunes A. Study of the liver changes occurring in preeclampsia and their possible pathogenetic connection with acute fatty liver of pregnancy. Am J Gastroenterol 1996; 91:292-4.

8. Riely CA, Latham PS, Romero R, Duffy TP. Acute fatty liver of pregnancy: a reassessment based on observations in nine patients. Ann Intern Med 1987; 106:703-6.

9. Sibai BM, Ramadan MK, Chari RS, Friedman SA. Pregnancies complicated by HELLP syndrome (hemolysis, elevated liver enzymes and low platelets): subsequent pregnancy outcome and long term prognosis. Am J Obstet Gynecol 1995; 172:125-9.

10.Reyes $H$. The spectrum of liver and gastrointestinal disease seen in cholestasis of pregnancy. Gastroenterol Clin North Am 1992; 21:905-21.

11.Reyes H, Gonzalez MC, Ribalta J, et al. Prevalence of intrahepatic cholestasis of pregnancy in Chile. Ann Intern Med 1978; 88:487-93.

12.Amon E, Allen SR, Petrie RH, Belew JE. Acute fatty liver of pregnancy associated with preeclampsia: management of hepatic failure with post-partum liver transplantation. Am J Perinatol 1991; 8:278-9.

13.Kaplan MM. Acute fatty liver of pregnancy. N Engl J Med 1985; 313:367-70.

14.Rolfes DB, Ishak KG. Acute fatty liver of pregnancy: a clinicopathologic study of 35 cases. Hepatology 1985; 5:1149-58.
15. Reyes H, Sandoval L, Wainstein A, et al. Acute fatty liver of pregnancy: a clinical study of 12 episodes in 11 patients. Gut 1994; 35:101-6.

16.Usta IM, Barton JR, Amon EA, Gonzalez A, Sibai BM. Acute fatty liver of pregnancy: an experience in the diagnosis and management of fourteen cases. Am J Obstet Gynecol 1994; 171:1342-7.

17.Bacq Y. Acute fatty liver of pregnancy. Semin Perinatol 1998; 22:134-40.

18.Barton JR, Riely CA, Adamec TA, Shanklen DR, Khoury AD, Sibai BM. Hepatic histopathologic condition does not correlate with laboratory abnormalities in HELLP syndrome (hemolysis, elevated liver enzymes, and low platelet count). Am J Obstet Gynecol 1992; 167:1538-43.

19.Minakami H, Oka N, Sato T, Tamada T, Yasuda Y, Hirota N. Preeclampsia: a microvesicular fatty disease of liver? Am J Obstet Gynecol 1988; 159:1043-7.

20.Ockner SA, Brunt EM, Cohn SM, Krul ES, Hanto DW, Peters MG. Fulminant hepatic failure caused by acute fatty liver of pregnancy treated by orthotopic liver transplantation. Hepatology 1990; 11:59-64.

21.Bacq Y, Sapey T, Bréchot MC, Pierre F, Fignon A, Dubois F. Intrahepatic cholestasis of pregnancy: a French prospective study. Hepatology 1997; 26:358-64.

22. Reyes H, Simon FR. Intrahepatic cholestasis of pregnancy: an estrogen-related disease. Semin Liver Dis 1993; 13:289-301.

23.Vanjak D, Moreau R, Roche-Sicot J, Soulier A, Sicot C. Intrahepatic cholestasis of pregnancy and acute fatty liver of pregnancy. An unusual but favorable association? Gastroenterology 1991; 100:1123-5.

24.Rioseco AJ, Ivankovic MB, Manzur A, et al. Intrahepatic cholestasis of pregnancy: a retrospective case control study of perinatal outcome. Am J Obstet Gynecol 1994; 170:890-5.

25.Mazzella G, Rizzo N, Salzetta A, Iampieri R, Bovicelli L, Roda E. Management of intrahepatic cholestasis in pregnancy. Lancet 1991; 338:15945 . 